\title{
HENRI BERGSON. HISTORIA DE LA IDEA DE TIEMPO (ADRIANA ALFARO, LUZ NOGUEZ TRADS.). PAIDÓS: BARCELONA, 2018. 397p.
}

Sin lugar a dudas, la filosofía de Henri Bergson representa una de las propuestas más novedosas y vigentes en tematizaciones acerca de la libertad, el problema estético-ontológico de la creación, la teoría de la imagen y la constitución temporal de lo real (durée). Desde la publicación, en 1889, de su tesis doctoral Essai sur les données immédiates de la conscience, ${ }^{1}$ Bergson comienza a posicionarse como un pensador crítico respecto de la tradición filosófica europea, pero propositivo respecto del lugar y función que corresponde a la filosofía dentro de las sociedades.

Podemos notar que el desarrollo de la filosofía de Bergson se vio íntimamente determinado por el contexto socio-político dentro del cual desempeñó sus funciones como docente. Junto a la obra ya mencionada, Matière et mémoire (1896), L'évolution créatrice (1907) y Les deux sources de la morale et de la religion (1932) conforman los textos más importantes de la filosofía bergsoniana y en los que más claramente podemos notar su evolución.

La obra que presentamos se constituye como una pieza clave no solo para comprender el desarrollo interno de la filosofía de Bergson, sino también para iluminar el despliegue de la filosofía francesa y, más puntualmente, sus principales tesis fenomenológicas de la temporalidad.

Historia de la idea del tiempo fue el nombre del curso impartido por Bergson entre el 5 de diciembre de 1902 y el 8 de mayo de 1903 (un total de diecinueve sesiones) en el Collège de France. Estas clases vieron la luz por vez primera el año 2016 gracias a la publicación de Presses Universitaires de France. ${ }^{2}$ Como es sabido, Bergson solicitó expresamente que, tras su muerte, no se publicara ni reprodujera ningún material que él no hubiera autorizado en vida (Bergson 2012, 1670). Es decir, el valor de la presente publicación consiste en que es un material absolutamente inédito, que además aporta elementos, perspectivas y consideraciones filosóficas fundamentales para la filosofía bergsoniana. En su presentación, Camille Riquier, editor del original, afirma que "los cursos publicados aquí no solo marcan el

${ }^{1}$ Cabe recordar que en 1889 Bergson presentó dos tesis para la defensa de su grado en la École Normale Supérieure: Essai sur les données inmédiates de la conscience y Quid Aristoteles de loco senserit.

2 Bergson, Henri. 2016. Histoire de l'idée de temps. Cours au Collège de France 1902 1903. Paris: PUF. 
inicio de 'la gloria de Bergson', la cual se extendió hasta la década de 1930, sino que, más aún, son su propia fuente" (Bergson, 15).

Es preciso señalar que las lecciones actualmente publicadas formaron parte de una serie de cursos que impartió Bergson en el Collège de France entre 1900 y 1914. Puntualmente, el presente curso fue precedido por "La idea de causa" (1900-1901) y "La idea de tiempo" (1901-1902) y es anterior a "Historia de las teorías de la memoria" (1903-1904) y "La evolución del problema de la libertad" (1904-1905).

Si prestamos atención, notaremos que las temáticas que aborda Bergson en sus cursos, de algún modo, muestran las temáticas generales que interesaron al filósofo francés y sintetizan los cambios que adoptará, sobre todo, desde Matière et mémoire (1896) hasta L'évolution créatrice (1907).

Ahora bien, respecto a las sesiones que dan forma a Historia de la idea del tiempo, es preciso destacar que, como se trata de una lectura históricofilosófica sobre la idea de tiempo, Bergson recurre constantemente a los textos originales de pensadores como Parménides, Platón, Aristóteles, Plotino y Galileo. En todos estos casos, el nobel francés ha realizado la traducción de los textos desde su idioma original al francés en el momento mismo de la clase. En la presente edición, dichos textos se han vertido al español desde la traducción francesa realizada por Bergson.

El desarrollo de las sesiones se estructura según el progreso histórico de las principales teorías filosóficas acerca del tiempo. De esta forma, las primeras cuatro sesiones son introductorias, y en ellas se destacan elementos presentados en sus cursos anteriores (sobre la idea de causa y la idea de tiempo).

En estas primeras sesiones, Bergson expone y articula su batería conceptual y argumentativa que, de hecho, le sirve como puente para el despliegue de las siguientes sesiones. Allí notamos que, sin señalarlo expresamente, Bergson vuelve a su primer trabajo al señalar que "conocer relativamente es conocer desde fuera, es estar fuera de lo que se aprende [...]. Conocer absolutamente [es conocer] desde dentro [...] no desde mí, sino desde ella misma, en sí" (Bergson, 27).

En efecto, para Bergson, el conflicto de lo temporal y los errores en los que ha caído una gran parte de la historia de la filosofía radica justamente en la modalidad por la cual interactuamos y conocemos el mundo. "Conocer relativamente es conocer desde fuera y conocer absolutamente es conocer desde dentro" (Bergson, 28). En el primer caso, solo podemos conocer por perfiles, por completitud perceptiva, lo que nos impide coincidir esencial- 
mente con lo otro; en el segundo caso, experimentamos la existencia absoluta de lo otro siendo sí mismo, es decir, desde su propia dignidad de existencia. Una bella cita de Édouard Le Roy, uno de sus alumnos y colegas más destacados, ilustra de manera precisa la relación entre el modo de conocer y la temporalidad bergsoniana:

El filósofo no debe mantenerse a la distancia de las cosas, sino practicar en ellas una especie de auscultación íntima y, sobre todo, ser capaz de ese esfuerzo de simpatía por medio del cual uno se instala en el objeto, se mezcla amigablemente con él, se pone de acuerdo con su ritmo original y, en una palabra, lo vive. (Le Roy, 38)

Lo que Bergson presenta en estas sesiones introductorias es la tematización metodológica de la simpatía intelectual (Bergson 29). Simpatizar intelectualmente significa conocer internamente lo otro, lo cual es posible gracias a que existe una tensión temporal que constituye toda realidad posible. De allí que "el problema de la duración es el problema central de la metafísica" (Bergson, 49).

Las sesiones no solo muestran lecturas críticas de Platón, Aristóteles y Descartes, sino que, por sobre todo, exponen las falencias y limitaciones de las teorías cientificistas que hegemonizaron la filosofía durante la transición del siglo XIX al XX. De hecho, al final de la segunda sesión Bergson afirma: "Nuestra ciencia no es capaz de alcanzar el interior; al menos, a través de sus métodos actuales, probablemente nunca lo lograría. Esto es algo que concierne a la metafísica" (Bergson, 60). Las palabras de Bergson coinciden con las primeras preocupaciones de la fenomenología de Husserl, quien un año antes había publicado Logische Untersuchungen, obra fundamental para el desarrollo de la filosofía fenomenológica.

En las siguientes sesiones, Bergson centrará su exposición en el problema que suscita la conceptualización y categorización de la temporalidad. Esto se debe a que "la esencia misma de la vida es la movilidad" (Bergson, 59). No obstante, el conocimiento conceptual es discontinuo y, por tanto, no logra aprehender con exactitud la continuidad de la realidad. Distendida y continua, por ser pura durée, la realidad solo es cognoscible desde su propia constitución temporal, no desde la conceptualización intelectual, ni por medio de un esquematismo categorial (Bergson, 86).

Ahora bien, si logro conocer de alguna manera al objeto, a lo otro, es porque no soy absolutamente exterior a ello. "Significa que la exterioridad recíproca de las cosas y de los seres es más bien una apariencia ordinaria de personalidad, o al menos artificial: algo necesario para la vida, porque la vida exige una separación, una especie de egoísmo entre los seres" (Bergson, 8788). 
Bergson iniciará su quinta sesión con el problema clásico eleático, que consiste en identificar el tiempo con un movimiento ejecutado dentro de un espacio absoluto de referencia. A juicio de Bergson, este modo de razonamiento, en la medida en que da inicio a la formalidad metafísica de razonamiento, cae en un problema metodológico fundamental. Este consiste en que, al focalizar la atención en el espacio inmóvil y absoluto dentro del cual se realiza el movimiento (y, por extensión, el devenir temporal), no se intenta pensar el movimiento o el devenir temporal, sino el espacio inmóvil y absoluto. Por ello Bergson sostiene que "debemos colocarnos en el movimiento mismo, no en lo inmóvil" (Bergson, 110), puesto que "no se puede obtener movimiento a partir de lo que es inmóvil" (Bergson, 111).

En relación con esto, Bergson señalará que la primera teoría propiamente filosófica sobre el tiempo se encuentra en el Timeo (Bergson, 113). A partir de aquí, las siguientes sesiones se ordenan como exposiciones generales de las principales teorías filosóficas del tiempo. Es justo señalar que el hecho de que sean exposiciones generales en ningún caso implica lecturas limitadas, sesgadas o erradas.

Los eleáticos se limitaron a negar el cambio; Platón quiso explicarlo, conservarlo, sosteniendo, sin embargo, que la realidad es lo inmutable, el concepto" (Bergson 120). Esta afirmación muestra el progreso de la filosofía platónica respecto de los eleáticos (Parménides y Zenón), pero también el límite en la teoría platónica acerca del tiempo: Platón "nunca se elevó a una concepción suficientemente abstracta de la causalidad como para imaginar la duración, el devenir, el tiempo, todo aquello, como algo que surgiera necesariamente de la eternidad, una vez que esta ha sido planteada (Bergson, 131).

Para Bergson, el mayor esfuerzo de abstracción sobre la temporalidad, como constituyente de la realidad, es realizado por Aristóteles. Por ello, dedica cuatro sesiones para abordar la tematización aristotélica sobre el tiempo, la forma y el problema de las causas (sesión séptima a la sesión décima). La radicalidad de esta exposición se sintetiza cuando el autor nos señala: "el movimiento no pudo haber comenzado ni terminado, porque si hubiera comenzado o terminado, habría un comienzo o un final del tiempo" (Bergson, 153). Esto se debe a que Aristóteles señala expresamente que el tiempo es una modalidad del movimiento, a diferencia de la tradición filosófica anterior a él, que concebía el movimiento como cualidad del tiempo.

A partir de la decimoprimera sesión, hacen aparición dos pensadores que influenciaron directamente la filosofía de Bergson, a saber: Plotino y Descartes. Respecto de Plotino, dirá que "es el primer y único filósofo antiguo que presenta una teoría de la conciencia” (Bergson, 221). La define, a la vez, como un estado interior de síntesis y un medio de análisis sobre el mundo. La teoría de la conciencia plotiniana es lo que animará a Bergson a sostener la posibilidad de comunicación y conocimiento de la vida (Bergson, 233). 
En relación con Descartes, Bergson continúa con el plan general de las sesiones anteriores, que consiste en exponer las dificultades que ha enfrentado la filosofía a lo largo de su historia respecto de los conflictos entre Dios y mundo, la inmutabilidad y el movimiento, o bien, la eternidad y el tiempo (Bergson, 153). Descartes habría partido desde la intuición y, por tanto, de la duración. No obstante, por su afán de encontrar una causa incondicionada, habría desplazado su punto de inicio "para buscar en una Idea pura [...] algo más sólido para respaldar su yo que huía en el tiempo" (Bergson, 340).

Además de los pensadores ya mencionados, en las exposiciones aparecen constantemente Galileo, Leibniz, Spinoza y Kant. Sin embargo, y tal como señala en la última sesión, a los tres últimos les dedica escuetos comentarios, dado que los cursos posteriores profundizarán en estos sistemas de pensamiento.

Historia de la idea del tiempo representa, como ya se señaló, una pieza clave para el transcurso y consolidación del pensamiento bergsoniano. Allí, en numerosas ocasiones encontramos un Bergson deudor de una enorme tradición filosófica, que, al mismo tiempo, va construyendo su filosofía propia, autónoma, contextualizada en su época y, por sobre todo, vigente tanto en profundidad conceptual, como en aportes metodológicos.

\section{BIBLIOGRAFIA}

Bergson, Henri. 2018. Historia de la idea del tiempo. Barcelona: Paidós.

Bergson. Henri. 2012. Correspondances. París: Presses Universitaires de France.

Le Roy, Édouard. 1932. Bergson. Madrid: Editorial Labor.

Sergio González Araneda Universidad de Santiago de Chile sgonzalezaraneda@gmail.com 